\title{
Peptide-functionalized quantum dots for potential applications in the imaging and treatment of obesity
}

This article was published in the following Dove Press journal:

International Journal of Nanomedicine

\author{
Ntevheleni Thovhogi' \\ Nicole Remaliah Samantha \\ Sibuyi' \\ Martin Opiyo Onani \\ Mervin Meyer' \\ Abram Madimabe Madiehe \\ 'Department of Science and \\ Technology (DST)/Mintek \\ Nanotechnology Innovation Centre, \\ Biolabels Unit, Department of \\ Biotechnology, ${ }^{2}$ Organometallics \\ and Nanomaterials, Department of \\ Chemistry, University of the Western \\ Cape, Bellville, South Africa
}

Background: Obesity is a worldwide epidemic affecting millions of people. The current pharmacological treatment of obesity remains limited and ineffective due to drugs' undesirable side effects. Hence, there is a need for novel or improved strategies for long-term therapies that will help prevent the disease progression into other chronic diseases. Nanotechnology holds the future for the treatment of obesity because of its versatility, as shown by improved drug efficiency and safety in cancer clinical trials. Nano-based drug delivery systems could potentially do the same for obesity through targeted drug delivery. This study investigated the use of peptide-functionalized quantum dots (QDs) for the imaging of prohibitin (PHB)-expressing cells in vitro and in diet-induced obese rats, which could potentially be used as nanocarriers of antiobesity drugs.

Methods: Cadmium (Cd)-based QDs were functionalized with an adipose homing peptide (AHP) and injected intravenously into lean and obese Wistar rats. Biodistribution of the QDs was analyzed by an IVIS ${ }^{\circledR}$ Lumina XR imaging system and inductively coupled plasma optical emission spectroscopy (ICP-OES). For in vitro studies, PHB-expressing (Caco-2 and MCF-7) and non-PHB-expressing (KMST-6 and $\mathrm{CHO}$ ) cells were exposed to either unfunctionalized QDs (QD625) or AHP-functionalized QDs (AHP-QD625) and analyzed by fluorescence microscopy.

Results: AHP-QD625 accumulated significantly in PHB-expressing cells in vitro when compared with non-PHB-expressing cells. In vivo data indicated that QD625 accumulated mainly in the reticuloendothelial system (RES) organs, while the AHP-QD625 accumulated mostly in the white adipose tissues (WATs).

Conclusion: AHP-functionalized QDs were successfully and selectively delivered to the PHBexpressing cells in vitro (Caco-2 and MCF-7 cells) and in the WAT vasculature in vivo. This nanotechnology-based approach could potentially be used for dual targeted drug delivery and molecular imaging of adipose tissues in obese patients in real time.

Keywords: adipose homing peptide, drug delivery, nanotechnology, prohibitin, white adipose tissue

\section{Introduction}

Obesity is a complex metabolic disease resulting from an excessive fat accumulation in the white adipose tissue (WAT). ${ }^{1-3}$ It is a major public health concern in both industrialized and developing countries and affects an estimated 1.4 billion of adult population. ${ }^{1}$ Obesity is a risk factor for the development of chronic diseases, such as diabetes, cardiovascular diseases, stroke, hypertension, and cancer. ${ }^{3,4}$ Obesity is characterized by an increase in the number (hyperplasia) and size (hypertrophy) of adipocytes in the WAT, which occurs when energy intake chronically exceeds energy expenditure. ${ }^{4,5}$ The WAT is an endocrine organ and known to secrete biologically 
active adipokines. The adipokines play a central role in WAT homeostasis, through the regulation of energy balance, insulin action, glucose metabolism, vascular remodeling, and angiogenesis..$^{5-8}$ Due to constant remodeling and expansion of the WAT, the tissue is highly vascularized and angiogenesis can serve as a target for therapeutic intervention. ${ }^{9,10}$

Angiogenesis refers to the formation of new blood vessels from the existing microvessels. It is a physiological process that occurs mainly during tissue growth, expansion, and repair. ${ }^{7-9}$ In obesity, the increase in the WAT mass is associated with increased angiogenesis ${ }^{10,11}$ and inhibition of this process has been shown to reverse obesity and its effects in animal models. ${ }^{5-12}$ Conventional treatment of obesity involves physical activity, healthy diet, pharmacotherapy, and surgery. ${ }^{1,7}$ However, pharmacological management of obesity is hampered by systemic drug toxicity and undesirable side effects. ${ }^{7}$ Therefore, there is an urgent need for the development of safer medication for the treatment of obesity. Nanotechnology emerges as a highly promising field that offers a novel means for solving the toxicity issues associated with the conventional antiobesity drugs. Nanotechnology involves the use of small particles at an atomic, molecular, and macromolecular scale. ${ }^{13}$ Various nanoparticles, functionalized with different biomolecules, have been successfully used in clinical and research applications for therapy due to their versatility, ease of chemical synthesis, low toxicity, and unique biophysical properties. ${ }^{14}$ Nanoparticles such as quantum dots (QDs) and gold nanoparticles (AuNPs) have been used in biomedical research, especially for molecular imaging and drug delivery, respectively. ${ }^{13,15-17}$ QDs are inorganic fluorescent semiconductor nanoparticles used as labeling and imaging tools in biomedical research. ${ }^{18-20}$ Unlike organic fluorophores and fluorescent proteins, QDs have a broad excitation spectra, narrow emission spectra, and long fluorescence half-life and can be conjugated to biomolecules, making them excellent probes for bio-imaging applications. ${ }^{17-20}$ The optical properties of QDs are attributed to quantum confinement due to their nanoscale size. ${ }^{17}$ Moreover, their high fluorescence quantum yield and resistance to photo bleaching make them good fluorescent labeling agents for targeted delivery and cellular imaging. ${ }^{16,17}$

The current study reports on molecular imaging and targeted delivery of QDs to prohibitin (PHB) expressed by the endothelial cells (ECs) in the WAT of obese subjects. The adipose homing peptide (AHP) was conjugated to cadmium (Cd)-based QDs for selective targeting and imaging of PHB both in vitro and in vivo. AHP (amino acid sequence: CKGGRAKDC) was discovered by Kolonin et $\mathrm{al}^{21}$ through phage display and shown to bind with high specificity to PHB expressed in the WAT vasculature of obese mice, ${ }^{21,22}$ rats, ${ }^{23}$ and monkeys. ${ }^{24} \mathrm{PHB}$ is a multifunctional membrane protein that has been used as a vascular marker of adipose tissue growth in obesity..$^{21,22,24,25}$ Targeting of a proapoptotic peptide to PHB expressed in the WAT vasculature caused ablation of white fat of leptin-deficient $\left(L e p^{o b / o b}\right)$ mice and reduced their body weight. ${ }^{21} \mathrm{We}$ also demonstrated uptake, binding, and specificity of AHP-targeted AuNPs in the WATs of obese rats as a plausible strategy for antiobesity-targeted drug delivery system. ${ }^{23}$ In parallel, the current study investigated bio-imaging of PHB-targeted QDs and this strategy could be used for the diagnosis and prognosis of obesity and monitoring the response to the treatment.

\section{Methods \\ Conjugation of QDs to AHP}

The $\operatorname{dot}^{\circledR} 625$ streptavidin-conjugate (QD625) was purchased from Thermo Fisher Scientific (Waltham, MA, USA), and the biotinylated AHP peptide was purchased from Anaspec (Fremont, CA, USA). The QD625 was conjugated to AHP through biotin-streptavidin chemistry to produce AHP-QD625, and the two peptides were mixed together to a final concentration of $25 \mathrm{nM}$ in $1 \times$ phosphate-buffered saline (PBS; pH 7.4). Both the functionalized (AHP-QD625) and nonfunctionalized (QD625) QDs were characterized by using agarose gel electrophoresis and a NanoLog FL3-22TRIAX spectrofluorometer (Horiba, Edison, NJ, USA).

\section{In vitro studies evaluating the binding of QDs to cells}

Human (Caco-2, MCF-7, and KMST-6) and Chinese Hamster Ovary (CHO) cell lines were purchased from American Type Culture Collection (ATCC) (Manassas, VA, USA). Caco-2 (colorectal adenocarcinoma), MCF-7 (breast carcinoma), and KMST-6 (skin fibroblast) cells were cultured in Dulbecco's Modified Eagle's Medium (Lonza, Verviers, Belgium), while $\mathrm{CHO}$ cells were cultured in Ham-F-12 media (Lonza) supplemented with $10 \%$ fetal bovine serum (Biochrom, Cambourne, UK) and $0.5 \%$ penicillin/streptomycin (Lonza). The cells were seeded on cover slips ( $5 \mathrm{~mm}$ diameter) at a cell density of $2 \times 10^{5}$ cells $/ \mathrm{mL}$ in a six-well plate and cultured for $24 \mathrm{~h}$ at $37^{\circ} \mathrm{C}$ in a humidified incubator. The cells were fixed with $4 \%$ paraformaldehyde for $10 \mathrm{~min}$ and then rinsed with PBS. The cells were subsequently incubated with either AHPQD625 (50 nM) or QD625 (50 nM), prepared in their respective media for $1 \mathrm{~h}$ at $37^{\circ} \mathrm{C}$. The coverslips were then mounted on microscope slides using Fluoroshield ${ }^{\mathrm{TM}}$ (Sigma-Aldrich 
Co., St Louis, MO, USA) with diamidino-2-phenylindole (DAPI). The slides were viewed under a Zeiss Axioplan 2 fluorescence microscope at $100 \times$ magnification.

\section{Induction of obesity}

Three-week-old male Wistar rats $(\mathrm{n}=16)$ were obtained from the South African Medical Research Council (SAMRC) Primate Unit and housed under a controlled temperature, humidity, ventilation, and a $12 \mathrm{~h}$ light/dark cycle. All procedures involving animals were approved by the SAMRC Ethics Committee for Research on Animals (ECRA) (Ref 03/10), and the protocol was conducted according to the guidelines of the ECRA. The rats were divided into two groups, the low fat (LF) and the high fat (HF) groups. The LF group $(n=4)$ was fed standard rodent chow and the HF group $(n=16)$ was fed HF diet to induce obesity, as described previously. ${ }^{23}$ Water was provided ad libitum. The body weights of the rats were monitored weekly for 16 weeks.

\section{Isolation of primary microvascular ECs}

ECs were isolated from subcutaneous (SQ) and epididymal (Epi) WAT obtained from LF- and HF-fed male Wistar rats following a protocol by Seo et $\mathrm{al}^{26}$ with slight modifications. Briefly, $5 \mathrm{~g}$ of the WATs was washed in PBS and minced into $1-2 \mathrm{~mm}$ pieces. The tissues were enzymatically digested with $1 \mathrm{mg} / \mathrm{mL}$ of collagenase at $37^{\circ} \mathrm{C}$ for $1 \mathrm{~h}$ then filtered through $200 \mu \mathrm{m}$ nylon filters to separate the microvascular fraction from the adipocytes. The microvascular fraction was incubated with Dynabeads ${ }^{\circledR}$ CD31 (Thermo Fisher Scientific) for $20 \mathrm{~min}$ at $4{ }^{\circ} \mathrm{C}$ to isolate $\mathrm{ECs}$, following manufacturer's instructions. The EC-bound beads were captured using the DynaMag ${ }^{\mathrm{TM}}-2$ Magnet (Thermo Fisher Scientific) and washed three times with PBS. The viable EC cell density was determined using Trypan Blue exclusion assay on the Countess ${ }^{\circledR}$ Automated Cell Counter (Thermo Fisher Scientific). The cells were used immediately for the QDs' cellular uptake experiment.

\section{AHP-QDs' binding and cellular uptake by primary ECs} The binding and uptake of AHP-QD625 to ECs was quantified by inductively coupled plasma optical emission spectroscopy (ICP-OES) following a previous protocol. ${ }^{23,27}$ Briefly, ECs at a concentration of $1 \times 10^{6} \mathrm{cells} / \mathrm{mL}$ were incubated for $1 \mathrm{~h}$ at $37^{\circ} \mathrm{C}$ in a humidified incubator with $50 \mathrm{nM}$ of either QD625 or AHP-QD625. The cells were washed twice with PBS, lysed with aqua regia $\left(3 \mathrm{HCl}: 1 \mathrm{HNO}_{3}\right)$ and digested at $90^{\circ} \mathrm{C}$ for $2 \mathrm{~h}$. The samples were diluted in $2 \% \mathrm{HNO}_{3}$ and analyzed using the 710-ES ICP-OES instrument (Varian, Palo Alto, CA, USA), and Cd was used as a standard.

\section{Quantification of QDs' distribution in tissues}

After 16 weeks of obesity induction, the HF rats were body weight matched into three HF groups $(n=4)$. The rats were singly caged for the duration of the study. On the day of QD administration, the rats were deeply anesthetized by nasal inhalation of halothane (initiation at 5\% and maintenance at $1 \%$ in $100 \%$ oxygen at $1 \mathrm{~L} / \mathrm{min}$ flow rate). The rats were injected intravenously with functionalized AHPQD625 (50 nM), unfunctionalized QD625 (50 nM), or PBS (untreated). Twenty-four hours postinjection, the rats were sacrificed by exsanguination under anesthesia. Blood sample was collected through the hepatic portal vein. Various tissues (WATs) and organs (liver, lungs, kidneys, spleen, stomach, brain, testes, pancreas, heart, and selected WAT) were dissected, weighed, and imaged using the IVIS ${ }^{\circledR}$ Lumina XR (Caliper Life Sciences, Waltham, MA, USA). After imaging, the tissues were washed with PBS and then stored at $-80^{\circ} \mathrm{C}$ until further analysis. The blood samples were stored at $4^{\circ} \mathrm{C}$.

The tissues and organs were processed according to previously published procedures ${ }^{28}$ with some modifications. One gram of WAT and of each organ was cut into small pieces, homogenized, and completely lysed in aqua regia in glass bottles. The residue was digested at $70^{\circ} \mathrm{C}$ in an oven for $48 \mathrm{~h}$. The residue was then re-dissolved in $2 \mathrm{~mL}$ of $2 \%$ $\mathrm{HCl}$ and sonicated for 2-5 min or until re-suspended. The resultant samples were diluted in $2 \% \mathrm{HNO}_{3}$ and analyzed by ICP-OES as described previously.

\section{Statistics}

Different parameters were analyzed using a one-way analysis of variance (ANOVA), followed by a Student's $t$-test. All the analyses were performed using the GraphPad Prism 5 statistical package. The samples were considered statistically significant when $P<0.05$.

\section{Results and discussion}

The study investigated the targeted delivery, specificity, and binding of AHP-QDs to the cells and tissues that express PHB. The study also explored the potential use of these nanoparticles for the imaging of PHB expressing tissues. The distribution of the PHB-targeted QDs was assessed in vitro, ex vivo and in vivo. In vitro studies investigated the uptake of Cd-based QDs in four cell lines, of which Caco-2 cells were previously shown to express $\mathrm{PHB}$ as a cell surface receptor, while MCF-7 cells expressed PHB in the cytosol. ${ }^{23,27}$ The binding of AHP-QDs to microvascular primary ECs isolated from the WAT samples obtained from diet-induced obese Wistar rats was also examined. 
AHP-QDs' binding to PHB-expressing cells PHB-expressing (MCF-7 and Caco-2) and non-PHB expressing (CHO and KMST-6) cells were exposed to either QD625 or AHP-QD625, and binding of these nanoparticles was studied by fluorescent microscopy. A significant cellular binding and uptake of AHP-QD625 by MCF-7 and Caco-2 cells was observed as shown in Figure 1A. The red fluorescence signal represents uptake of the QDs by the cells, which is seen only on MCF-7 and Caco-2 cells exposed to AHP-QD625. No fluorescence was detected when the two cell lines were incubated with QD625 (untargeted QDs).

There was no indicative red fluorescence observed on CHO and KMST-6 cells exposed to both QD625 and AHPQD625, an indication that no cellular binding or uptake by these cells occurred. These data suggest that the mechanism for intracellular uptake of the targeted QDS by MCF-7 and Caco-2 cells was mediated by the PHB receptor. Based on the water-soluble tetrazolium-1 assay results, the QDs at $\leq 50 \mathrm{nM}$ were not toxic to all the cells used in the study for the duration of the experiment (data not shown).

\section{Binding and uptake of AHP-QDs by primary microvascular ECs from adipose tissues}

Microvascular ECs were isolated from Epi and SQ adipose tissues obtained from lean (LF) and obese (HF) rats. The cells were exposed to QD625 and AHP-QD625, and accumulation or uptake of $\mathrm{Cd}$ metal was analyzed by ICP-OES. Figure 2 shows the binding and uptake of QDs by the ECs isolated from Epi and SQ. ECs from lean rats (LF), exposed to QD625 and AHP-QD625, showed reduced levels of Cd in both Epi and SQ. In contrast, ECs from the HF group treated with AHP-QD625 showed significantly higher Cd levels in the two tissues. This suggested that QD625 and AHP-QD625 were differentially bound and/or taken up by ECs obtained from diet-induced obese rats than their lean counterparts. Moreover, the binding of AHP-QD625 was significantly higher in HF rats and indicates a receptor-mediated binding and uptake. This could be due to an increased PHB expression on the cell surface of ECs from obese rats, ${ }^{23} \mathrm{PHB}$ was shown to be upregulated in the plasma membrane of vascular ECs in the WATs of obese subjects. ${ }^{21-25}$

\section{In vivo studies}

To determine whether the observed in vitro effects of QDs can be replicated in vivo, male Wistar rats were fed either an LF diet or a HF diet for 16 weeks to induce obesity. The rats (LF and HF group) received a nontoxic amount of $50 \mathrm{nM}$ of either QD625 or AHP-QD625 for $24 \mathrm{~h}$. The obese rats on the HF diet had a significantly higher body weight (701.2 $\pm 14.8 \mathrm{~g}$ ) compared to the lean rats, which were on an LF diet $(539.7 \pm 15.9 \mathrm{~g})$. Figure 3 shows the selected animal body tissue and organ weights of lean and obese rats at the termination of the study. The adipose tissue (SQ, Epi, mesenteric [Mes], retroperitoneal [Retro], and perirenal [Peri]) weights in the HF group were also higher than those in the LF group. The weight differences observed for the other organs were not statistically significant for the two groups.

\section{Imaging and biodistribution of QDs in vivo}

The obese (HF) rats were intravenously injected with PBS (untreated control), QD625, or AHP-QD625 and sacrificed after $24 \mathrm{~h}(\mathrm{n}=4)$. Various organs and tissues were dissected to assess the biodistribution of QDs ex vivo. The organs and tissues from the three HF groups were visualized using an IVIS ${ }^{\circledR}$ Lumina XR and then quantified by ICP-OES. Figures $4 \mathrm{~A}$ and $5 \mathrm{~A}$ show the representative image analysis of QDs' fluorescent signal in selected organs and WATs of obese rats injected with PBS (control), QD625, or AHPQD625. High fluorescent intensities of unfunctionalized QD625 were observed mainly in the liver, kidneys, testes, and brain, as shown by the intense yellow fluorescence signal, but were rarely distributed in the WATs. Epi exhibited relatively low levels of fluorescent signal of QD625 and barely noticeable in the other WATs (Figure 5A). In contrast, the fluorescent signal of AHP-QD625 was significantly higher in the WATs but not in the liver, testes, kidneys, and brain. No fluorescence was observed in organs from rats injected with PBS (untreated).

Figures $4 \mathrm{~B}$ and $5 \mathrm{~B}$ show the quantitative analysis of $\mathrm{Cd}$ content in the tissues of rats injected with both the unfunctionalized QD625 and functionalized AHP-QD625. High concentrations of $\mathrm{Cd}$ accumulated mostly in the reticuloendothelial system (RES) organs (liver, lungs, spleen, and kidneys) of rats injected with QD625 (Figure 4B), while low amounts of Cd were found in the WATs (Figure 5B). In contrast, the rats injected with QDs that contained the PHB targeting peptide (AHP-QD625) showed increased concentrations of $\mathrm{Cd}$ in the WATs and the Cd levels were significantly reduced in the liver, lungs, spleen, kidneys, and testes. These findings are in accordance with previously reported studies, ${ }^{15,29,30}$ where most of the unfunctionalized nanoparticles accumulated in the RES organs (liver, lungs, kidneys, and spleen). These results also indicate that AHP-QD625 accumulates preferentially in the WATs, further corroborating studies showing that AHP targets PHB on the WAT vasculature of obese animals. $^{21-25}$ 


\section{A}
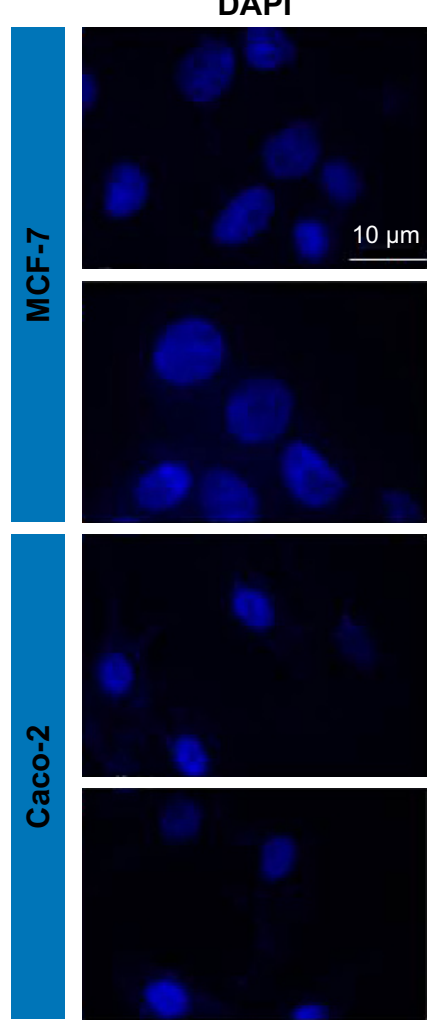

B
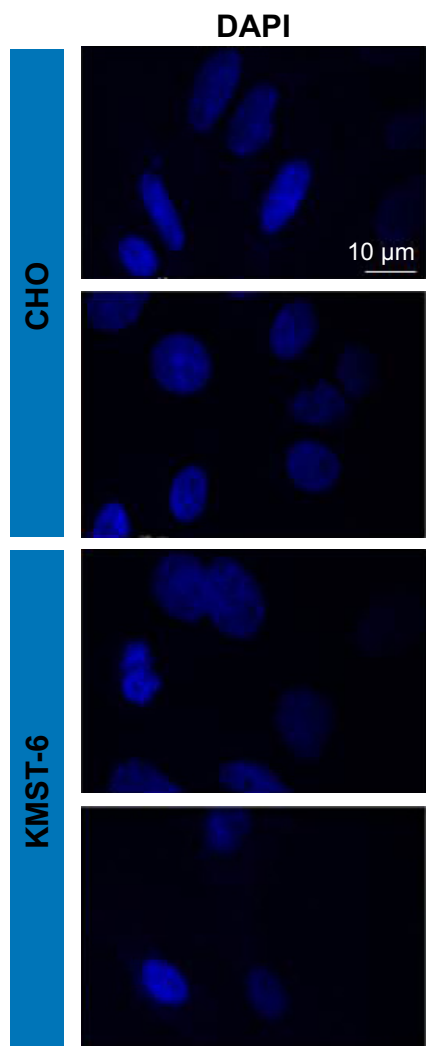

QDs
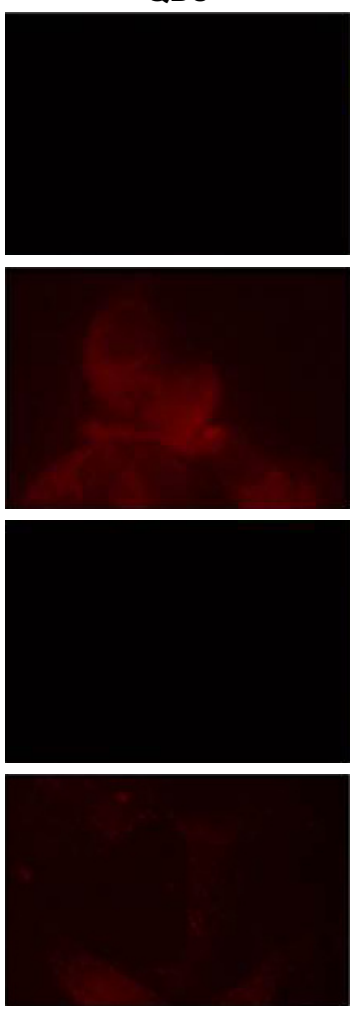

QDs
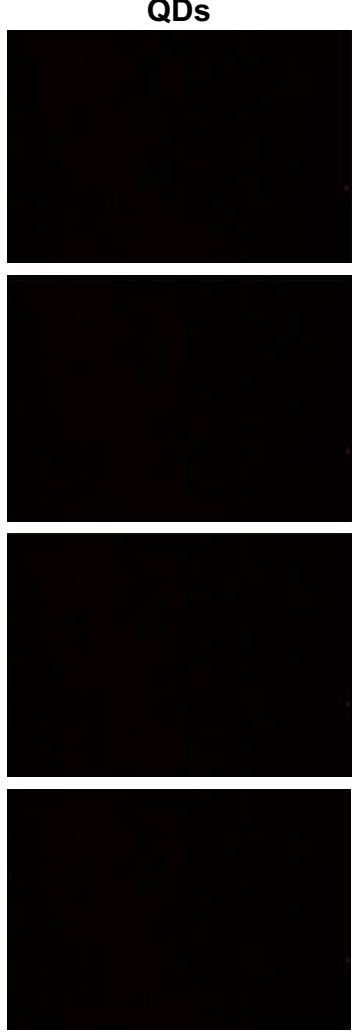

\section{Overlay}
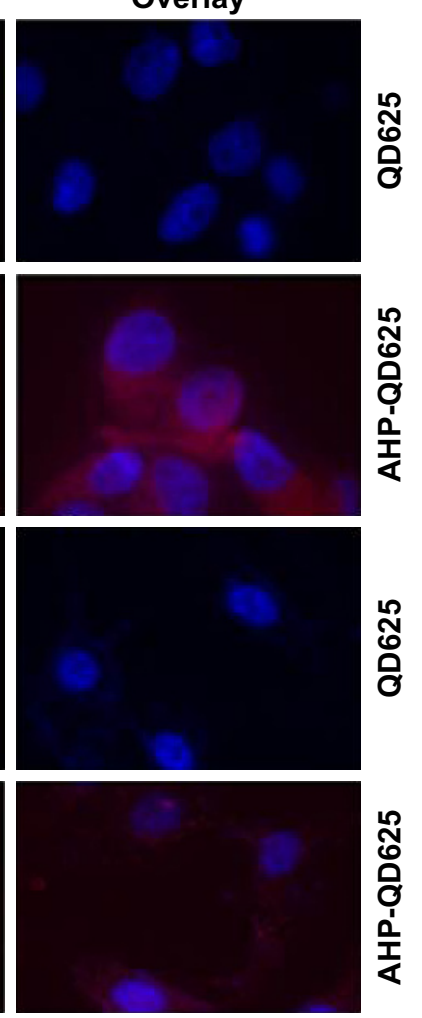

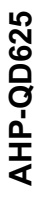
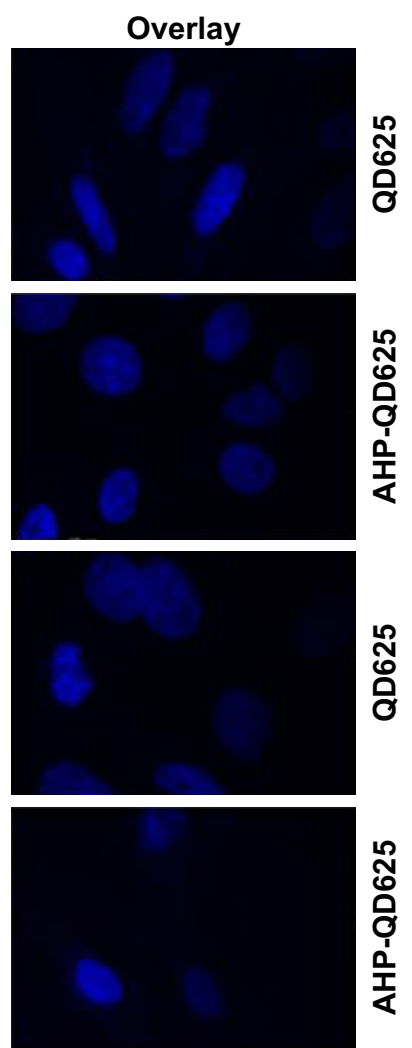

Figure I Fluorescence imaging of cells treated with QD625 or AHP-QD625.

Notes: PHB-expressing (A) and non-PHB expressing (B) cells were treated with QD625 or AHP-QD625 for I h; the images were captured using a fluorescence microscope. Scale bars, $10 \mu \mathrm{m}$. Blue, nuclei stain (DAPI) and red, QDs' fluorescence.

Abbreviations: AHP, adipose homing peptide; DAPI, diamidino-2-phenylindole; PHB, prohibitin; QDs, quantum dots. 

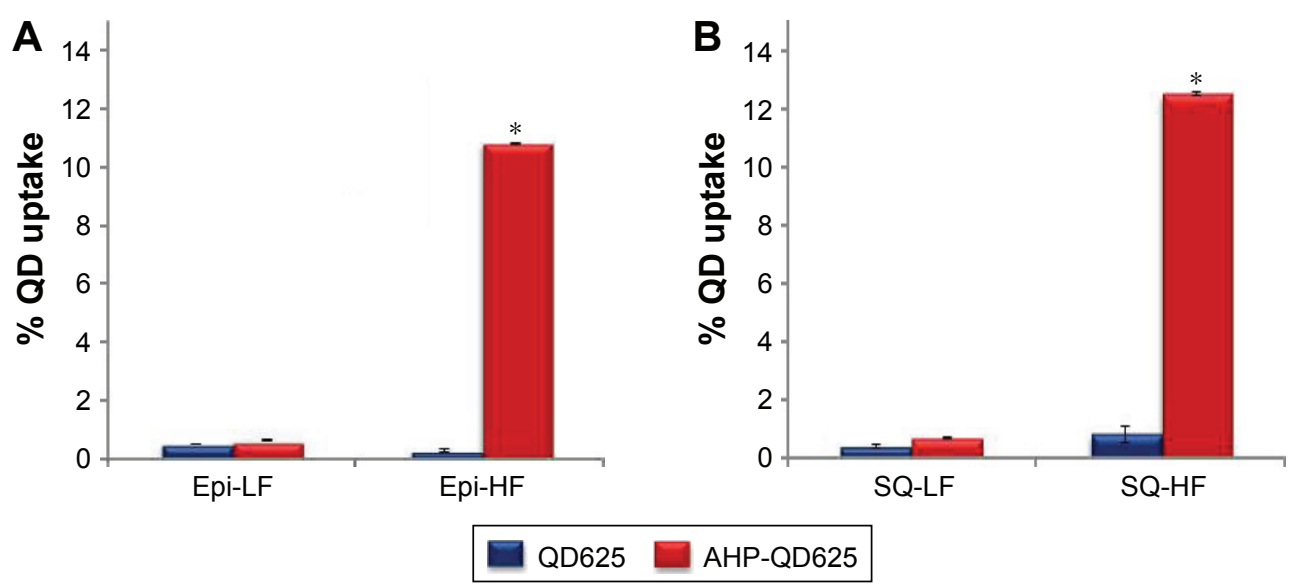

Figure 2 Cellular binding and uptake of QD625 and AHP-QD625 by microvascular ECs isolated from the WATs.

Notes: ECs isolated from Epi (A) and SQ (B) WATs of lean and obese rats were treated with QD625 and AHP-QD625 for I h at $37^{\circ} \mathrm{C}$. Bar graphs show Cd as a percentage of the treatment dose present in each sample as determined by ICP-OES analysis. $n=3$; *statistically significant at $P<0.05$.

Abbreviations: AHP, adipose homing peptide; Cd, cadmium; ECs, endothelial cells; Epi, epididymal; HF, high fat; ICP-OES, inductively coupled plasma optical emission spectroscopy; LF, low fat; SQ, subcutaneous; QD, quantum dot; WATs, white adipose tissues.

In the current study, the QDs served as fluorescence probes for cellular and in vivo imaging of PHB-expressing cells. The QDs proved to be biocompatible and stable and targeted the PHB receptor when conjugated to AHP (Figures 4 and 5). Biodistribution and targeted delivery of PHB-targeted QDs were reproducible in vitro (Figure 1), ex vivo (Figure 2), and in vivo (Figures 4 and 5). Similar data were reported on the use of PHB-targeted AuNPs in vitro and in vivo. ${ }^{23}$ Although the toxicity of $\mathrm{Cd}$ is irrefutable, ${ }^{31}$ the uptake and toxicity of the Cd-based QDs is dependent on their size, shape, charge, shell, and concentration. ${ }^{32-34}$ These parameters can be modified and customized for clinical applications, by attaching biomolecules such as PEG to passivate the nanomaterials. Conjugation of biomolecules to the QD surface can potentially reduce leaching of the toxic Cd metal from the core ${ }^{34,35}$ and assist in the clearance of the QDs through renal filtration. ${ }^{32,33}$ An independent study demonstrated that skin-derived mesenchymal stem cells (MSCs) can be used as a vehicle for the transportation and selective delivery of $\mathrm{CdSe} / \mathrm{ZnS}$ QDs to cancer cells in vivo. ${ }^{36}$ These MSCs are known to selectively migrate and target wounds, inflammatory sites, and tumor cells..$^{36,37}$ QD-loaded skin MSCs were selectively delivered to cancer cells in human tumor xenograft models. ${ }^{36}$ While the fate of Cd-QDs still needs to be addressed, the QDs' excellent properties can come in handy in assays where toxicity is not an issue such as ELISAs, immunocytochemistry for cellular imaging. ${ }^{34}$ Meanwhile, other less toxic QDs such as indium-based QDs can be considered for live imaging in vivo. ${ }^{38}$

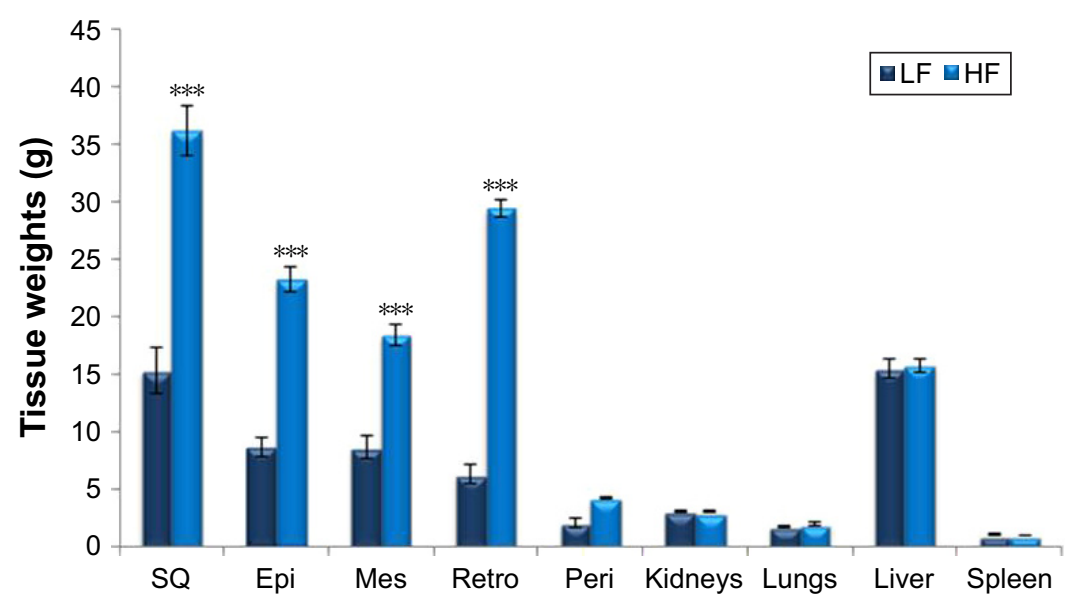

Figure 3 Body and tissue weights of LF- and HF-fed rats measured at termination.

Notes: ***Statistically significant at $P<0.000$ I. Results are presented as mean $\pm S E M, n=20$.

Abbreviations: Epi, epididymal; HF, high fat; LF, low fat; Mes, mesenteric; Peri, perirenal; Retro, retroperitoneal; SQ, subcutaneous. 

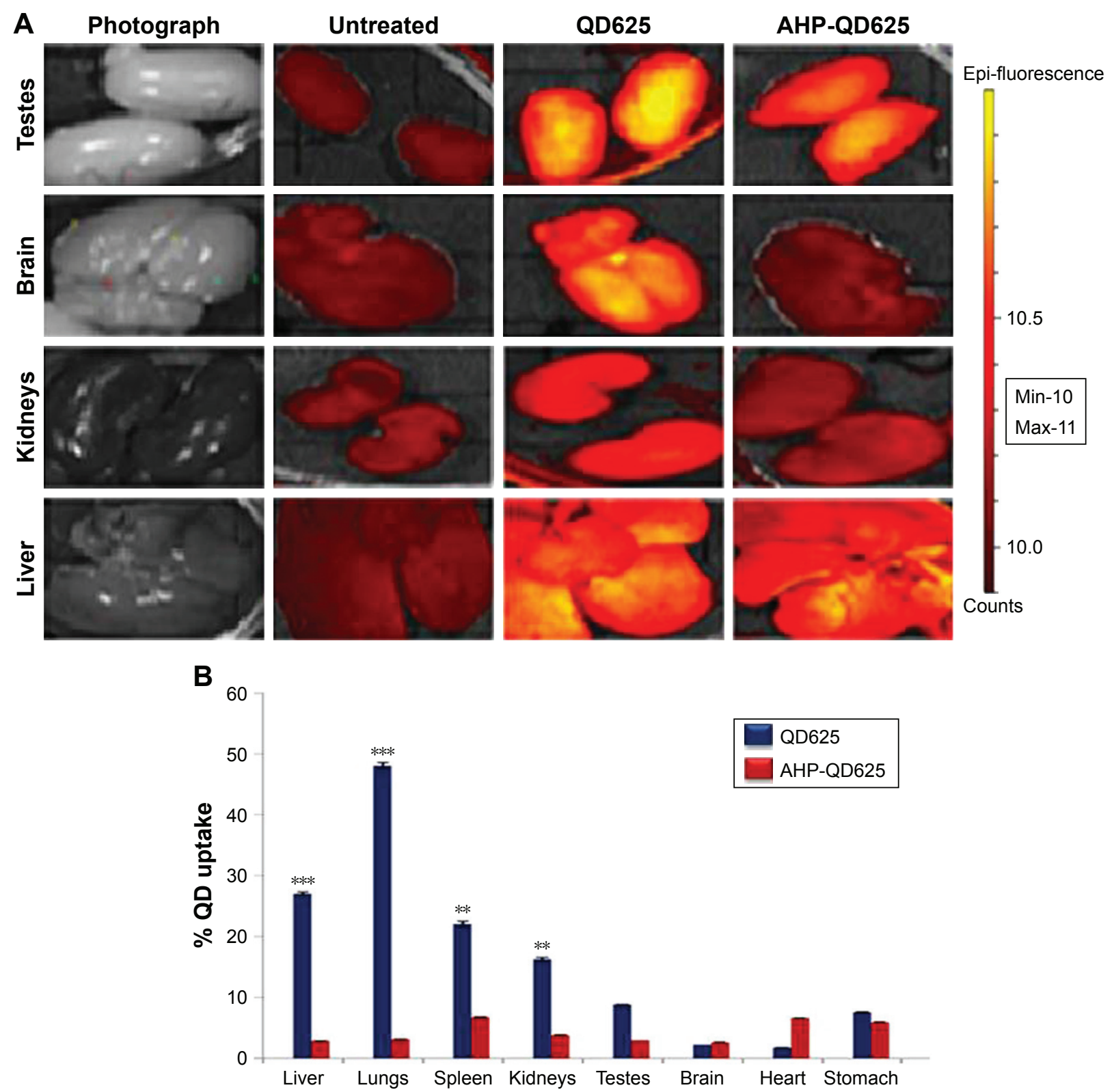

Figure 4 Fluorescent imaging and quantitative biodistribution of QDs in organs isolated from obese Wistar rats.

Notes: The rats were intravenously injected with PBS (untreated), QD625, and AHP-QD625. Fluorescence images of selected organs were acquired using IVIS ${ }^{\circledR}$ Lumina XR (A). Yellow color, QDs' fluorescence. Bar graphs show Cd as a percentage of the injected dose present in each sample as determined by ICP-OES analysis (B). ** Statistically significant at $P<0.001 ; * * * P<0.0001$.

Abbreviations: AHP, adipose homing peptide; Cd, cadmium; Epi, epididymal; ICP-OES, inductively coupled plasma optical emission spectroscopy; Max, maximum; Min, minimum; PBS, phosphate-buffered saline; QD, quantum dot.

\section{Conclusion}

The study demonstrated the selective delivery and imaging of AHP-QD625 on PHB-expressing (Caco-2 and MCF-7) cells and the WAT vasculature of obese Wistar rats. This might represent a potential nanotechnology-based approach for targeted drug delivery and live imaging systems of pathological adipose tissue and could also be used to determine the efficacy of antiobesity agents during the treatment of obesity. The study showed the use of biocompatible QDs for targeting and imaging of cells and tissue vasculature that express PHB in vitro, ex vivo, and in vivo. However, the potential toxicity of Cd-based QDs due to Cd remains a concern. ${ }^{20}$ Moreover, due to the short duration of the experiments in this study, a determination on whether the persistence of $\mathrm{Cd}$ might produce long-term toxicity was not made. Therefore, it is important to study whether long-term exposure to Cd-based QDs has toxic effects on the animals. The use of less toxic QDs for live imaging purposes should be considered in the future. 

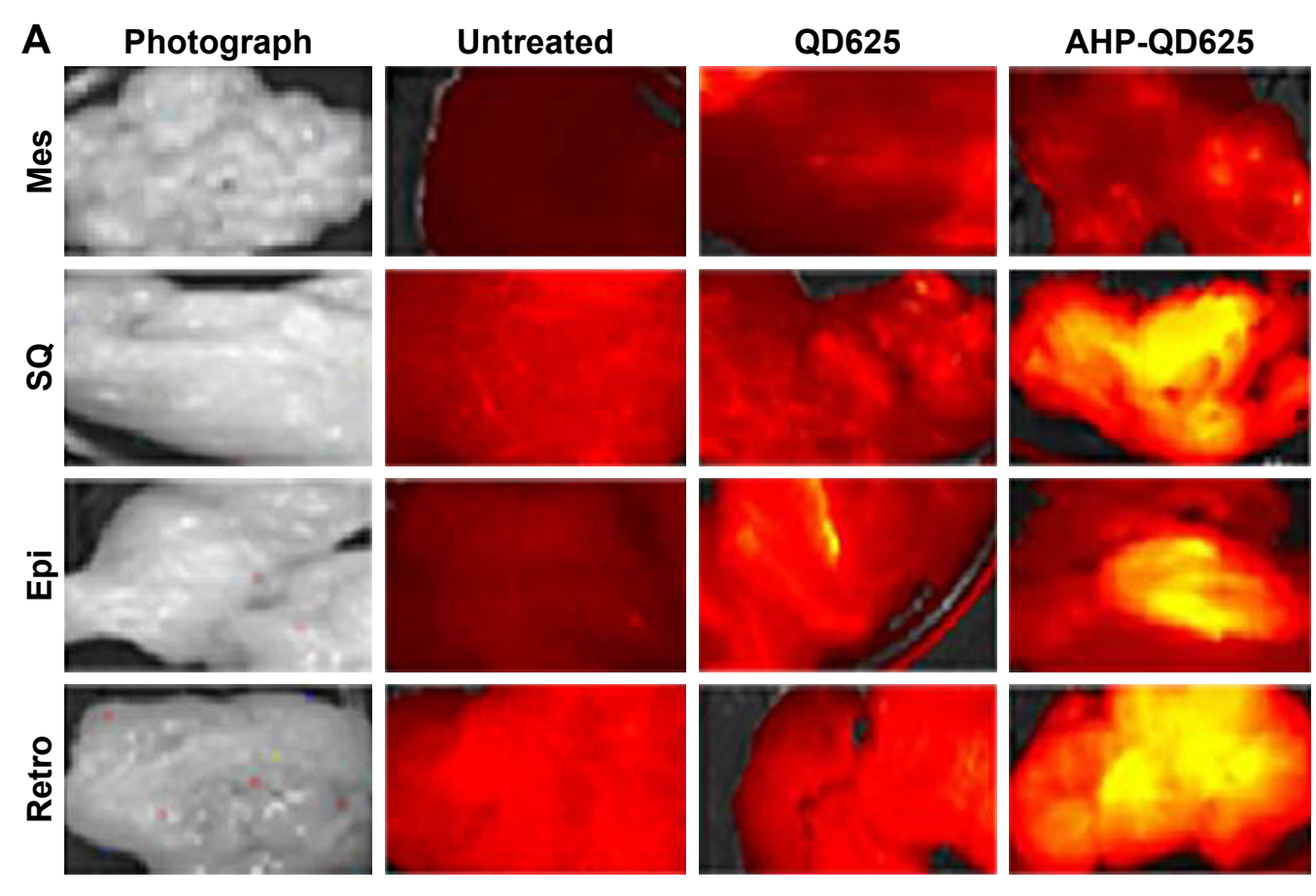

Epi-fluorescence
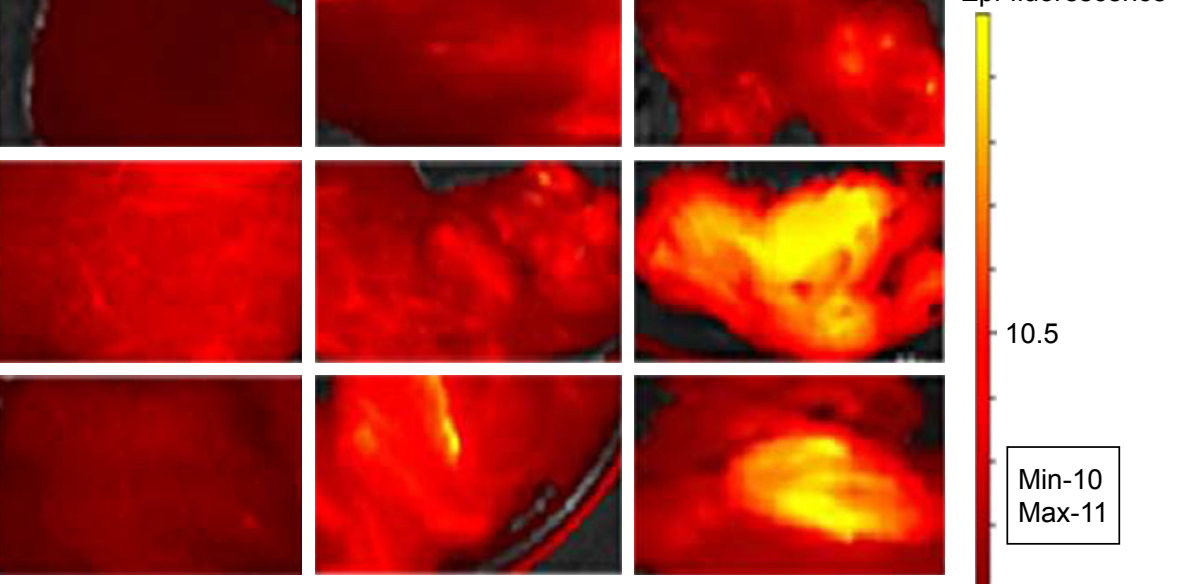

10.5
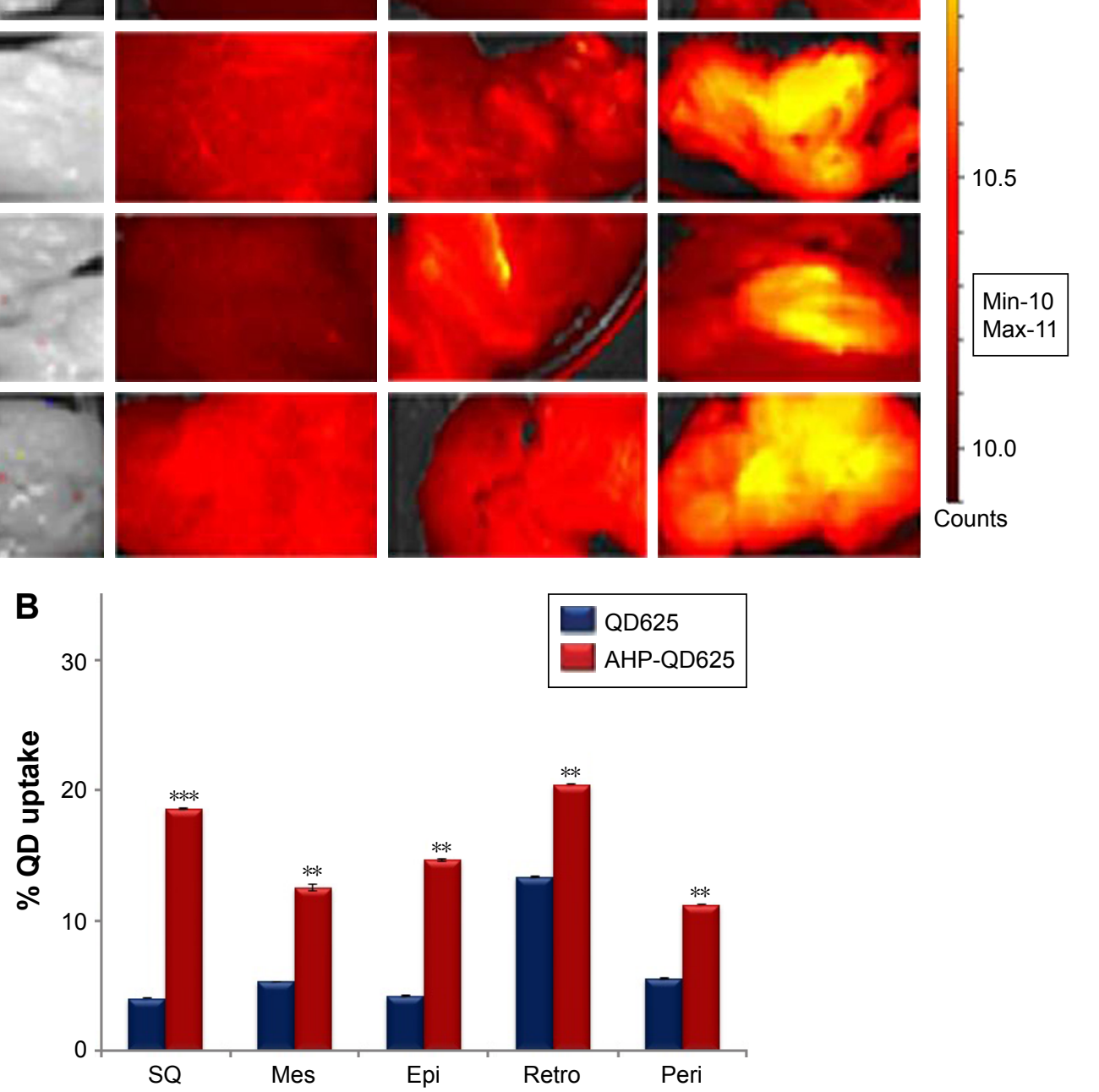

Figure 5 Fluorescent imaging and quantitative biodistribution of Cd-based QDs in WATs isolated from obese Wistar rats, $24 \mathrm{~h}$ postinjection.

Notes: The rats were intravenously injected with PBS (untreated), QD625, or AHP-QD625. Fluorescence images of selected organs were acquired using IVIS ${ }^{\circledR}$ Lumina $\mathrm{XR}(\mathbf{A})$. Yellow color $=\mathrm{QDs}$ ' fluorescence. Bar graphs show $\mathrm{Cd}$ as a percentage of the injected dose present in each sample as determined by ICP-OES analysis $(\mathbf{B})$. $\mathrm{n}=3$; ***statistically significant at $P<0.001$; ***P $<0.0001$.

Abbreviations: AHP, adipose homing peptide; Cd, cadmium; Epi, epididymal; ICP-OES, inductively coupled plasma optical emission spectroscopy; Max, maximum; Mes, mesenteric; Min, minimum; PBS, phosphate-buffered saline; Peri, perirenal; QD, quantum dot; Retro, retroperitoneal; SQ, subcutaneous; WATs, white adipose tissues.

\section{Acknowledgment}

This study was funded by grants from the South African Department of Science and Technology (DST)/Mintek Nanotechnology Innovation Centre, National Research Foundation and SAMRC.

\section{Disclosure}

The authors report no conflicts of interest in this work.

\section{References}

1. Zimmerman-Belsing T, Feldt-Rasmusson U. Obesity: the new worldwide epidemic threat to general health and our complete lack of effective treatment. Endocrinology. 2004;145:1501-1502.
2. Kissler HJ, Settmacher U. Bariatric surgery to treat obesity. Semin Nephrol. 2013;33(1):75-89.

3. Spalding KL, Arner E, Westermark PO, et al. Dynamics of fat cell turnover in humans. Nature. 2008;453(7196):783-787.

4. Prieto-Hontoria PL, Pérez-Matute P, Fernández-Galilea M, Bustos M, Martínez JA, Moreno-Aliaga MJ. Role of obesity-associated dysfunctional adipose tissue in cancer: a molecular nutrition approach. Biochim Biophys Acta. 2011;1807(6):664-678.

5. Daquinag AC, Zhang Y, Kolonin MG. Vascular targeting of adipose tissue as an anti-obesity approach. Trends Pharmacol Sci. 2011;32(5): 300-307.

6. Cao Y. Adipose tissue angiogenesis as a therapeutic target of obesity and metabolic diseases. Nat Rev Drug Discov. 2010;9:107-115.

7. Cao Y. Angiogenesis modulates adipogenesis and obesity. J Clin Invest. 2007;117(9):2362-2368.

8. Cao Y. Angiogenesis as a therapeutic target for obesity and metabolic diseases. Chem Immunol Allergy. 2014;99:170-179. 
9. Xue Y, Xu X, Zhang XQ, Farokhzad OC, Langer R. Preventing dietinduced obesity in mice by adipose tissue transformation and angiogenesis using targeted nanoparticles. Proc Natl Acad Sci U S A. 2016; 113(20):5552-5557.

10. Rupnik MA, Panigrahy D, Zhang CY, et al. Adipose tissue mass can be regulated through the vasculature. Proc Natl Acad Sci U S A. 2002; 99(16):10730-10735.

11. Lijnen HR. Angiogenesis and obesity. Cardiovasc Res. 2007;78(2): 286-293.

12. Lemoine AY, Ledoux S, Larger E. Adipose tissue angiogenesis in obesity. Thromb Haemost. 2013;110(4):661-668.

13. Raghavendra R, Arunachalam K, Annamalai SK, Arunachalam AM. Diagnostics and therapeutic application of gold nanoparticles. Int $J$ Pharm Sci. 2014;6:74-87.

14. Bobo D, Robinson KJ, Islam J, Thurecht KJ, Corrie SR. Nanoparticlebased medicines: a review of FDA-approved materials and clinical trials to date. Pharm Res. 2016;33(10):2373-2387.

15. Papasani MR, Wang G, Hill RA. Gold nanoparticles: the importance of physiological principles to devise strategies for targeted drug delivery. Nanomedicine. 2012;8(6):804-814.

16. Zhang H, Yee D, Wang C. Quantum dots for cancer diagnosis and therapy: biological and clinical perspectives. Nanomedicine (Lond). 2008; 3(1):83-91.

17. Barroso MM. Quantum dots in cell biology. J Histochem Cytochem. 2011;59(3):237-251.

18. Mulder WJ, Strijkers GJ, Nicolay K, Griffioen AW. Quantum dots for multimodal molecular imaging of angiogenesis. Angiogenesis. 2010; 13(2):131-134.

19. Kim MW, Jeong HY, Kang SJ, et al. Cancer-targeted nucleic acid delivery and quantum dot imaging using EGF receptor aptamerconjugated lipid nanoparticles. Sci Rep. 2017;7(1):9474.

20. Tang J, Huang N, Zhang X, et al. Aptamer-conjugated PEGylated quantum dots targeting epidermal growth factor receptor variant III for fluorescence imaging of glioma. Int J Nanomedicine. 2017;12 3899-3911.

21. Kolonin MG, Saha PK, Chan L, Pasqualini R, Arap W. Reversal of obesity by targeted ablation of adipose tissue. Nat Med. 2004;10(6):625-632.

22. Hossen MN, Kajimoto K, Akita H, Hyodo M, Ishitsuka T, Harashima H Ligand-based targeted delivery of a peptide modified nanocarrier to endothelial cells in adipose tissue. J Control Release. 2010;147(2): 261-268.

23. Thovhogi N, Sibuyi N, Meyer M, Onani M, Madiehe A. Targeted delivery using peptide-functionalised gold nanoparticles to white adipose tissues of obese rats. J Nanopart Res. 2015;17:112.
24. Barnhart KF, Christianson DR, Hanley PW, et al. A peptidomimetic targeting white fat causes weight loss and improved insulin resistance in obese monkeys. Sci Transl Med. 2011;3(108):108ra112.

25. Hossen MN, Kajimoto K, Akita H, Hyodo M, Harashima H. Vasculartargeted nanotherapy for obesity: unexpected passive targeting mechanism to obese fat for the enhancement of active drug delivery. J Control Release. 2012;163(2):101-110.

26. Seo MJ, Suh SY, Bae YC, Jung JS. Differentiation of human adipose stromal cells into hepatic lineage in vitro and in vivo. Biochem Biophys Res Commun. 2005;328(1):258-264.

27. Sibuyi NRS, Thovhogi N, Gabuza KB, et al. Peptide-functionalized nanoparticles for the selective induction of apoptosis in target cells. Nanomedicine. 2017;12(14):1631-1645.

28. Sonavane G, Tomoda K, Makino K. Biodistribution of colloidal gold nanoparticles after intravenous administration: effect of particle size. Colloids Surf B Biointerfaces. 2008;66(2):274-280.

29. Lasagna-Reeves C, Gonzalez-Romero D, Barria MA, et al. Bioaccumulation and toxicity of gold nanoparticles after repeated administration in mice. Biochem Biophys Res Commun. 2010;393(4): 649-655.

30. Morais T, Soares ME, Duarte JA, et al. Effects of surface coating on the biodistribution profile of gold nanoparticles in rats. Eur J Pharm Biopharm. 2011;80:185-193.

31. Bernhoft RA. Cadmium toxicity and treatment. Scientific World Journal. 2013;2013:394652.

32. Choi HS, Liu W, Misra P, et al. Renal clearance of quantum dots. Nat Biotechnol. 2007;25(10):1165-1170.

33. Galdiero E, Falanga A, Siciliano A, et al. Daphnia magna and Xenopus laevis as in vivo models to probe toxicity and uptake of quantum dots functionalized with gH625. Int J Nanomedicine. 2017;12: 2717-2731.

34. Zhang LW, Monteiro-Riviere NA. Mechanisms of quantum dot nanoparticle cellular uptake. Toxicol Sci. 2009;110(1):138-155.

35. Rzigalinski BA, Strobl JS. Cadmium-containing nanoparticles: perspectives on pharmacology and toxicology of quantum dots. Toxicol Appl Pharmacol. 2009;238(3):280-288.

36. Dapkute D, Steponkiene S, Bulotiene D, Saulite L, Riekstina U, Rotomskis R. Skin-derived mesenchymal stem cells as quantum dot vehicles to tumors. Int J Nanomedicine. 2017;12:8129-8142.

37. Uchibori R, Tsukahara T, Ohmine K, Ozawa K. Cancer gene therapy using mesenchymal stem cells. Int J Hematol. 2014;99(4):377.

38. Kiplagat A, Sibuyi NRS, Onani MO, Meyer M, Madiehe AM. The cytotoxicity studies of water-soluble InP/ZnSe quantum dots. $J$ Nanopart Res. 2016;18:147.
International Journal of Nanomedicine

\section{Publish your work in this journal}

The International Journal of Nanomedicine is an international, peerreviewed journal focusing on the application of nanotechnology in diagnostics, therapeutics, and drug delivery systems throughout the biomedical field. This journal is indexed on PubMed Central,

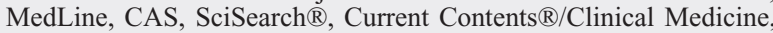

\section{Dovepress}

Journal Citation Reports/Science Edition, EMBase, Scopus and the Elsevier Bibliographic databases. The manuscript management system is completely online and includes a very quick and fair peer-review system, which is all easy to use. Visit http://www.dovepress.com/ testimonials.php to read real quotes from published authors. 